\title{
A Post Hoc Analysis of HbA1c, Hypoglycemia, and Weight Change Outcomes with Alogliptin vs Glipizide in Older Patients with Type 2 Diabetes
}

Morgan Bron • Craig Wilson · Penny Fleck

To view enhanced content go to www.diabetestherapy-open.com

Received: August 26, 2014 / Published online: November 26, 2014

(C) The Author(s) 2014. This article is published with open access at Springerlink.com

\section{ABSTRACT}

Introduction: Adverse events and complications limit the long-term use of current antidiabetic treatment options for patients with type 2 diabetes mellitus (T2DM), particularly for older adults who are often receiving therapy for other comorbid conditions. The aim of this study was to evaluate the benefits of the dipeptidyl peptidase-4 inhibitor, alogliptin, versus glipizide, a sulfonylurea, in achieving glycemic control without the risk of hypoglycemia, weight gain, or both in older patients with T2DM.

Methods: This was an exploratory, post hoc analysis of a global, multicenter, randomized,

Trial registration: ClinicalTrials.gov \#NCT00707993.

Electronic supplementary material The online version of this article (doi:10.1007/s13300-014-0088-5) contains supplementary material, which is available to authorized users.

M. Bron ( $\square)$

Takeda Pharmaceuticals International, One Takeda Parkway, Deerfield, IL 60015, USA

e-mail: morgan.bron-green@takeda.com

C. Wilson · P. Fleck

Takeda Global Research and Development Center, Inc., One Takeda Parkway, Deerfield, IL, USA double-blind, active-controlled study comparing alogliptin and glipizide. Patients $(n=441)$ aged $65-90$ years with glycosylated hemoglobin (HbA1c) 6.5-9.0\% who failed on diet and exercise alone or who had inadequately controlled T2DM despite oral antidiabetic monotherapy were recruited from 110 sites across 15 countries. Alogliptin $25 \mathrm{mg}(n=222)$ or glipizide $5 \mathrm{mg}$ up-titrated to $10 \mathrm{mg}(n=219)$ was administered once daily for 52 weeks. Composite endpoints of HbA1c $\leq 7.0 \%$ coupled with the absence of hypoglycemia and weight gain, or an HbA1c reduction of $\geq 0.5 \%$ in the absence of hypoglycemia and weight gain, were then measured.

Results: In the primary analysis, least squares mean HbA1c changes from baseline to Week 52 were similar in both the alogliptin and glipizide groups. The proportion of patients achieving HbA1c $\leq 7.0 \%$ without hypoglycemia or weight gain was significantly higher for alogliptin versus glipizide $(24 \%$ vs $13 \%, p<0.03)$. Patients with a baseline HbA1c of $<8.0 \%$ receiving alogliptin were also more likely to achieve HbA1c $\leq 7.0 \%$ without hypoglycemia or weight gain than those receiving glipizide $(29 \%$ vs $13 \%, p<0.03)$. 
Conclusion: Alogliptin demonstrated similar efficacy to glipizide in lowering $\mathrm{HbA1c}$ in older patients with T2DM, but with significantly more patients achieving an HbA1c $\leq 7.0 \%$ without hypoglycemia or an increase in body weight. These results particularly apply to patients with baseline HbA1c below 8.0\%.

Funding: The study was sponsored by Takeda Global Research \& Development Center, Inc., Deerfield, IL, and Takeda Global Research and Development Centre Ltd., London, United Kingdom.

Keywords: Alogliptin; Glipizide; HbA1c; Hypoglycemia; Type 2 diabetes mellitus; Weight

\section{INTRODUCTION}

Type 2 diabetes mellitus (T2DM) is very common in older individuals. Indeed, US data from the Centers for Disease Control and Prevention in 2011 showed that the percentage of diagnosed diabetes was more than 13 times higher in people aged 65-74 years when compared with those younger than 45 years of age; the overall percentages for these two populations were reported to be $21.8 \%$ and $1.6 \%$, respectively [1]. Furthermore, the most recent International Diabetes Federation Diabetes Atlas estimates that the global burden of diabetes in older individuals will increase greatly by 2030 (between $42 \%$ and $>50 \%$ ) in people over the age of 60 [2].

There are a number of treatment options available for patients with diabetes; however, many of them are associated with significant clinical adverse events (AEs). Metformin, which is considered the standard initial therapy for T2DM in conjunction with diet and exercise regimens [3], is associated with gastrointestinal AEs and, rarely, lactic acidosis [4]. It is also contraindicated in patients with compromised renal function: the package insert states that metformin treatment "should not be initiated in patients $\geq 80$ years of age unless measurement of creatinine clearance demonstrates that renal function is not reduced" [4], which is a common comorbid condition in older patients. Moreover, serum creatinine levels may be unreliable in an older patient with low muscle mass. Similarly, sulfonylureas, which are often prescribed as an initial therapy in patients who are not overweight, or as an additional therapy in patients who have failed to achieve adequate glycemic control with metformin alone, are associated with hypoglycemia and weight gain [3]. Hypoglycemia is of particular importance in older patients because drug-induced hypoglycemic episodes may go unrecognized and may be confounded by cognitive dysfunction. Hypoglycemic episodes are more common and often more serious in older patients receiving oral antidiabetic drugs and can lead to complications such as falls and hip fractures, as well as even more serious cardiovascular events and death $[5,6]$. These AEs and complications all limit the long-term use of sulfonylureas. This is particularly true in older adults who often have underlying health problems and may also be receiving treatment for other comorbid conditions.

Alogliptin is a dipeptidyl peptidase-4 (DPP-4) inhibitor that has shown efficacy as a treatment for T2DM, either as monotherapy in conjunction with diet and exercise or as an adjunct therapy to other diabetic treatments such as sulfonylureas, metformin, thiazolidinediones, or insulin [7-11]. In particular, in an exploratory retrospective pooled analysis of one phase 2 and five phase 
3 studies, alogliptin was shown to be consistently efficacious and demonstrated a good safety profile in patients aged between 65 and 80 years. These benefits were seen when alogliptin was administered both as monotherapy and as an add-on therapy, and was not found to increase the incidence of hypoglycemia, weight gain, or other AEs when compared with younger patients [6].

In this exploratory, post hoc analysis of a prospective study specifically designed to evaluate the efficacy of alogliptin and glipizide, a sulfonylurea, we focused on patients aged 65-90 years over a 52-week period [12]. The purpose of the study was to explore the benefits of alogliptin in achieving glycemic control without the risk of weight gain, hypoglycemia, or both, as compared with glipizide in this population of patients with T2DM.

\section{METHODS}

\section{Study Design}

This study was an exploratory post hoc analysis of data obtained from a global, multicenter, randomized, double-blind, active-controlled study (NCT 00707993) that evaluated the efficacy and safety of alogliptin versus glipizide in patients with T2DM between the ages of 65 and 90 years. Patients were recruited from 110 sites across 15 countries. To be included patients must have failed on diet and exercise alone in the 2 months prior to screening and had glycosylated hemoglobin (HbA1c) of $6.5-9.0 \%$ or had inadequately controlled T2DM (HbA1c 6.5-8.0\%) despite oral antidiabetic monotherapy, which had to be washed out during the lead-in period. Patients must have been able and willing to self-monitor blood glucose with a home glucose monitor [12].
Patients were included in a screening period of up to 2 weeks followed by a 52-week treatment period. At Week 52, patients underwent an end-of-study visit and a followup visit 2 weeks later. Treatment-naïve patients were immediately randomized while patients who had received oral monotherapy underwent a 4-week washout period and were randomized if their HbA1c level was between 6.5\% and 9.0\% without antidiabetic medication [12].

Patients were randomized to receive alogliptin (25 mg) or glipizide (5 mg) once daily. Glipizide could be titrated from 5 to $10 \mathrm{mg}$ in patients with persistent hyperglycemia. Post-titration dose reductions were allowed only once, mainly for hypoglycemia. Patients who continued to be hyperglycemic while receiving either study drug were administered rescue medication according to increasingly stringent rescue criteria once elevated fasting plasma glucose or HbA1c was confirmed [12].

The analysis in this article is based on previously conducted studies and does not involve any new studies of human or animal subjects performed by any of the authors. The original study was performed in accordance with the Declaration of Helsinki, the International Conference on Harmonisation's Harmonised Tripartite Guideline for Good Clinical Practice, and all applicable regional laws and regulations. An institutional review board or ethics committee conducted the initial approval and continuing review of the study and all patients signed an informed consent form prior to undergoing any procedures.

\section{Composite Endpoints}

The primary endpoint for the initial study was HbA1c changes at Week 52 from baseline using the last observation carried forward and the per- 
protocol set, consisting of all randomized and treated patients with no major protocol violations. Secondary endpoints included changes from baseline in HbA1c at all time points and by baseline $\mathrm{HbA1c}$, fasting plasma glucose, 2-h postprandial glucose, hyperglycemic rescue, and weight and lipid changes over time [12].

In this exploratory analysis, the composite endpoint of $\mathrm{HbA} 1 \mathrm{c} \leq 7.0 \%$ coupled with the absence of hypoglycemia and no body weight gain was analyzed. HbA1c reduction of $\geq 0.5 \%$ coupled with the absence of hypoglycemia and no weight gain was also assessed. All randomized and treated patients were used for these analyses. Hypoglycemic events were rigorously captured and identified using the stringent American Diabetes Association (ADA) Workgroup on Hypoglycemia-specified criteria of $<70 \mathrm{mg} / \mathrm{dL}$ ( $3.9 \mathrm{mmol} / \mathrm{L})$ [13]. Patients were provided with explicit instructions regarding the signs and symptoms of hypoglycemia at study entry. They then received diaries and blood glucose meters to self-report hypoglycemic episodes by recording glucose values and associated signs and symptoms. All glucose measurements were stored on the glucose meters, downloaded by the study personnel and included as part of the overall hypoglycemia evaluation. Hypoglycemic episodes were defined as plasma glucose below $70 \mathrm{mg} / \mathrm{dL}(3.9 \mathrm{mmol} / \mathrm{L})$ and were categorized as mild to moderate (symptomatic or asymptomatic) or severe (any episode requiring assistance from another person to actively administer carbohydrate, glucagon, or other resuscitative actions, regardless of whether plasma glucose was obtained).

\section{Safety}

Safety variables assessed in the original clinical study [12] included pretreatment events, AEs, clinical laboratory tests (hematology, serum chemistry, and urinalysis), vital sign measurements, physical examination findings, and 12-lead electrocardiography readings.

\section{Statistics}

All statistical analyses were performed in those who completed the study using SAS version 8.2 or higher (SAS Institute, Cary, NC, USA). Participants without a Week 52 HbA1c or weight value were treated as failures for the composite endpoints. All statistical tests were conducted at a 5\% significance level.

The composite endpoints were analyzed overall, irrespective of baseline HbA1c, using logistic regression models with a factor for treatment (alogliptin or glipizide) and with baseline HbA1c and baseline weight as covariates. Additional logistic regression models were fitted separately for patients with a baseline $\mathrm{HbA} 1 \mathrm{c}<8.0 \%$ and $\geq 8.0 \%$.

\section{RESULTS}

\section{Disposition, Demographics, and Baseline Characteristics}

The patient disposition of this population is shown in Fig. 1. A total of 441 patients were randomized: 222 patients to the alogliptin group and 219 to the glipizide group. In the alogliptin group, $60 \%$ completed the study, $25 \%$ received hyperglycemia rescue, and $15 \%$ discontinued for other reasons. In the glipizide group, the equivalent percentages were 57,22 , and $22 \%$, respectively. The mean age of the total population was 69.6 years and the mean baseline HbA1c was 7.5\%.

Patient demographics and baseline characteristics in the Randomized Set are described in Table 1. Overall, the patients in 


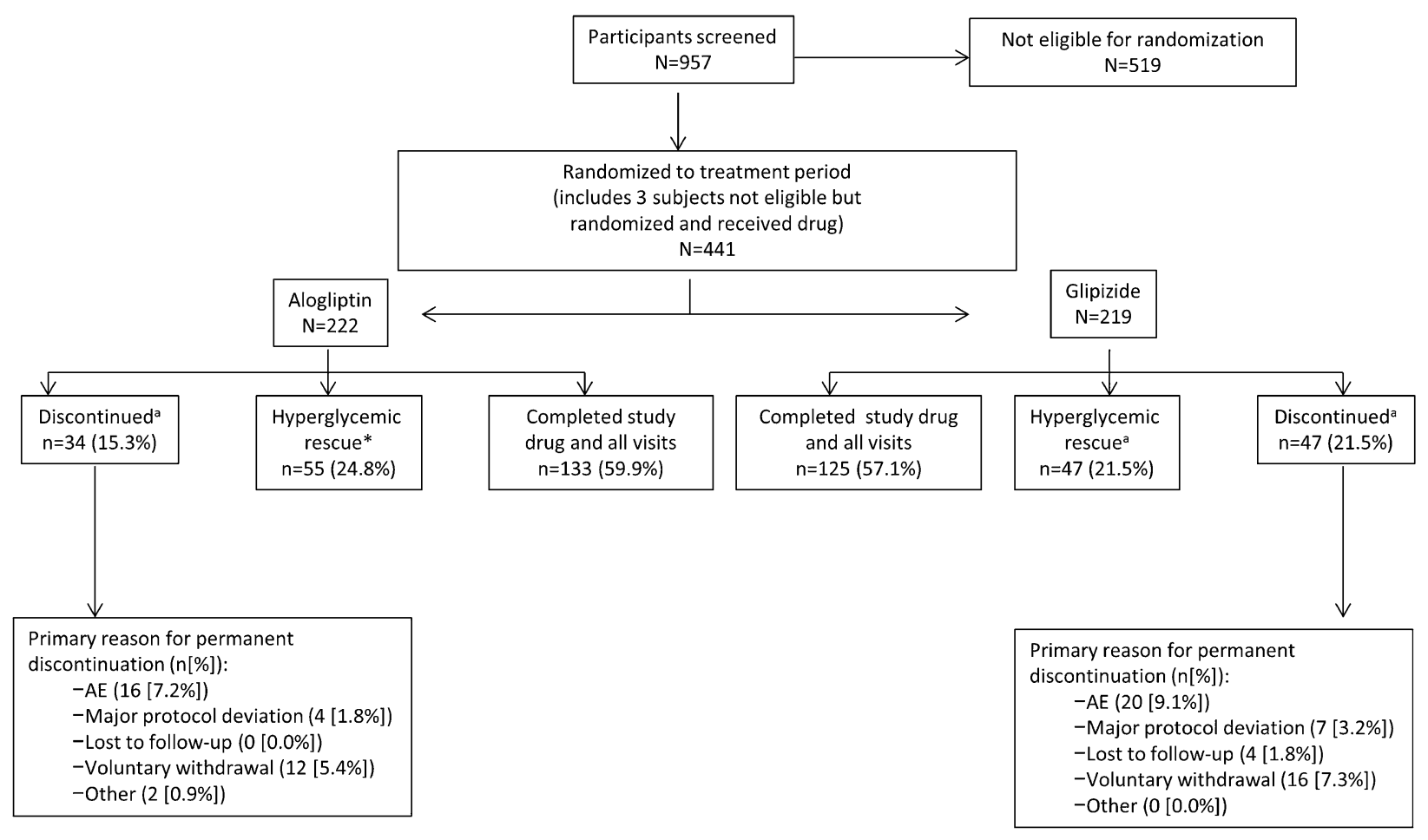

Fig. 1 Patient disposition of the study population (adapted from Rosenstock et al. [12]). ${ }^{\text {ac }}$ Hyperglycemic rescue” and "discontinued" were mutually exclusive groups. Participants who met the prespecified hyperglycemia rescue criteria and,

the alogliptin and glipizide groups were well matched for demographics and baseline characteristics. The majority of patients (approximately three-fourths in each group) had a baseline HbA1c of $<8.0 \%$. The mean \pm standard deviation (SD) body weights in the alogliptin and glipizide groups were $78.6 \pm 14.8 \mathrm{~kg}$ and $78.8 \pm 15.2 \mathrm{~kg}$, respectively, with mean $\pm \mathrm{SD}$ body mass index values of $29.6 \pm 4.4 \mathrm{~kg} / \mathrm{m}^{2}$ and $30.0 \pm 4.5 \mathrm{~kg} / \mathrm{m}^{2}$, respectively.

\section{Glycemic Control}

The results from the primary analysis in the original clinical study [12] showed that the least squares (LS) mean changes in HbA1c from baseline to Week 52 were similar between alogliptin and glipizide (Fig. 2). Indeed, the thus, discontinued treatment due to lack of efficacy were not included in the number of participants who discontinued therapy. $A E$ adverse event

reduction from baseline in LS mean observed HbA1c values was similar for both the alogliptin and glipizide groups throughout the 52-week study (Fig. 2).

An exploratory analysis of the per-protocol set, conducted in the primary study [12], revealed larger reductions in HbA1c among patients who completed the study $(-0.47 \%$ and $-0.31 \%$ with alogliptin and glipizide, respectively) than among rescued patients $(0.61 \%$ and $0.53 \%$ with alogliptin and glipizide, respectively). Similarly, greater HbA1c reductions occurred among drugnaïve patients $(-0.32 \%$ and $-0.12 \%$ with alogliptin and glipizide, respectively) than among the $45.6 \%$ of monotherapy patients who entered the study after a 4 -week washout $(0.09 \%$ and $-0.03 \%$ with alogliptin and glipizide, respectively). 
Table 1 Patient disposition, demographics and baseline characteristics in the randomized set

\begin{tabular}{|c|c|c|}
\hline Characteristic & $\begin{array}{l}\text { Alogliptin } \\
(N=222)\end{array}$ & $\begin{array}{l}\text { Glipizide } \\
(N=219)\end{array}$ \\
\hline \multicolumn{3}{|l|}{ Study populations, $n$ (\%) } \\
\hline Safety set & $222(100)$ & $219(100)$ \\
\hline Per-protocol set & $180(81)$ & $162(74)$ \\
\hline \multicolumn{3}{|l|}{ Geographic region, $n(\%)$} \\
\hline North America & $62(28)$ & $65(30)$ \\
\hline Latin America & $59(27)$ & $54(25)$ \\
\hline Europe, rest of the world & $101(46)$ & $100(46)$ \\
\hline \multicolumn{3}{|l|}{ Baseline HbAlc, $n(\%)$} \\
\hline$<8.0 \%$ & $168(76)$ & $170(78)$ \\
\hline$\geq 8.0 \%$ & $54(24)$ & $49(22)$ \\
\hline \multicolumn{3}{|l|}{ Sex, $n(\%)$} \\
\hline Male & $102(46)$ & $96(44)$ \\
\hline \multicolumn{3}{|l|}{ Age } \\
\hline Mean $\pm S D$, years & $70.1 \pm 4.4$ & $69.8 \pm 4.1$ \\
\hline $\begin{array}{l}\text { Median (minimum- } \\
\text { maximum), years }\end{array}$ & $\begin{array}{l}69.0 \\
(65-86)\end{array}$ & $\begin{array}{l}69.0 \\
\quad(65-87)\end{array}$ \\
\hline$<75$ years, $n(\%)$ & $186(84)$ & $193(88)$ \\
\hline$\geq 75$ years, $n(\%)$ & $36(16)$ & $26(12)$ \\
\hline \multicolumn{3}{|l|}{ Race, $n(\%)$} \\
\hline $\begin{array}{l}\text { American Indian or Alaskan } \\
\text { Native }\end{array}$ & $12(5)$ & $13(6)$ \\
\hline Asian & $19(9)$ & $26(12)$ \\
\hline Black or African American & $16(7)$ & $20(9)$ \\
\hline White & $169(76)$ & $154(70)$ \\
\hline Multiracial & $6(3)$ & $6(3)$ \\
\hline Mean \pm SD weight, $\mathrm{kg}$ & $78.6 \pm 14.8$ & $78.8 \pm 15.2$ \\
\hline Mean \pm SD BMI, $\mathrm{kg} / \mathrm{m}^{2}$ & $29.6 \pm 4.6$ & $30.0 \pm 4.5$ \\
\hline $\begin{array}{c}\text { Mean } \pm \text { SD diabetes } \\
\text { duration, years }\end{array}$ & $6.25 \pm 6.3$ & $5.94 \pm 6.3$ \\
\hline Mean \pm SD HbAlc, $\%(n)^{\mathrm{a}}$ & $\begin{array}{c}7.5 \pm 0.7 \\
(215)\end{array}$ & $\begin{array}{c}7.5 \pm 0.6 \\
(214)\end{array}$ \\
\hline
\end{tabular}

Table 1 continued

\begin{tabular}{lcl}
\hline Characteristic & $\begin{array}{l}\text { Alogliptin } \\
(\boldsymbol{N}=\mathbf{2 2 2})\end{array}$ & $\begin{array}{l}\text { Glipizide } \\
(\boldsymbol{N}=\mathbf{2 1 9})\end{array}$ \\
\hline Mean \pm SD GFR, $\mathrm{mL} / \mathrm{min} / 1.73 \mathrm{~m}^{2}$ & \\
MDRD & $74 \pm 15$ & $73 \pm 16$ \\
Cockcroft-Gault & $78.3 \pm 18$ & $78 \pm 20$ \\
\hline
\end{tabular}

$B M I$ body mass index, GFR glomerular filtration rate, HbAIc glycosylated hemoglobin, MDRD Modification of Diet in Renal Disease, $S D$ standard deviation

${ }^{a}$ Evaluated using all randomized and treated participants with available HbAlc data after baseline

\section{Composite Endpoints}

A significantly greater proportion of all patients in the alogliptin group achieved an HbA1c of $\leq 7.0 \%$ without hypoglycemia and weight gain compared with the glipizide group (Figs. 3, 4). Similar results were also observed when an HbA1c decrease of $\geq 0.5 \%$ was assessed, although the difference did not reach statistical significance (Figs. 3,4 ). In the cohort of patients with baseline HbA1c $<8.0 \%$, significantly higher percentages of patients in the alogliptin group achieved the target HbA1c of $\leq 7.0 \%$ and an HbA1c decrease of $\geq 0.5 \%$, both coupled with a lack of hypoglycemia and weight gain, compared with the glipizide group (Figs. 3, 4). Similar numbers of participants in the alogliptin and glipizide groups with baseline $\mathrm{HbA} 1 \mathrm{c} \geq 8.0 \%$ achieved the composite endpoints (Figs. 3, 4).

\section{Safety}

Full safety results from the original study have been reported previously [12]. In brief, a total of 314 patients $(71.2 \%)$ experienced one or more AEs. Most individual AEs occurred in fewer than $1 \%$ of patients overall. The most common $\mathrm{AE}$ associated with alogliptin was headache $(2.7 \%$, 


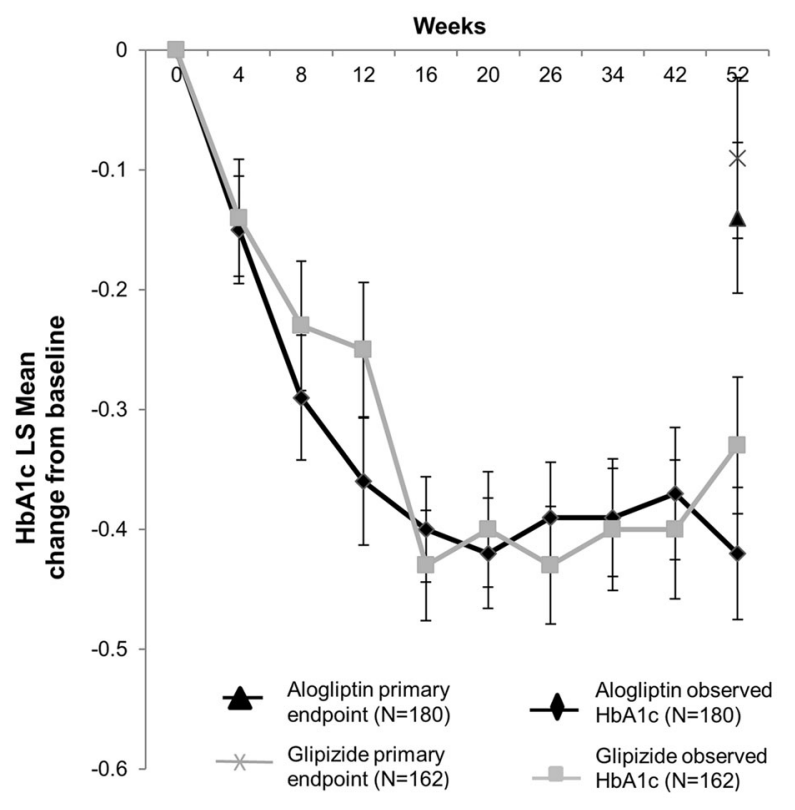

Fig. 2 Mean change in $\mathrm{HbAlc} \pm \mathrm{SE}$ (observed and primary endpoint) from baseline to Week 52 in the perprotocol set. $H b A 1 c$ glycosylated hemoglobin, $L S$ least squares, $S E$ standard error. Figure adapted from Rosenstock et al. [12]

with the same incidence also being reported for glipizide). Hypoglycemia and dizziness were the most common glipizide-associated AEs, with $3.7 \%$ of patients in the glipizide group reporting each of these events versus $0 \%$ and $0.9 \%$ in the alogliptin group, respectively.

\section{DISCUSSION}

Alogliptin demonstrated similar efficacy to glipizide in lowering $\mathrm{HbA1c}$ in this older population of patients with T2DM, as previously reported in the original clinical study [12]. This exploratory analysis has extended the data from the original study and demonstrated that significantly more patients in the alogliptin group achieved an HbA1c goal of $\leq 7.0 \%$ without hypoglycemia and weight gain when compared with the glipizide group. In general, in this older population of patients with T2DM, alogliptin was well tolerated and
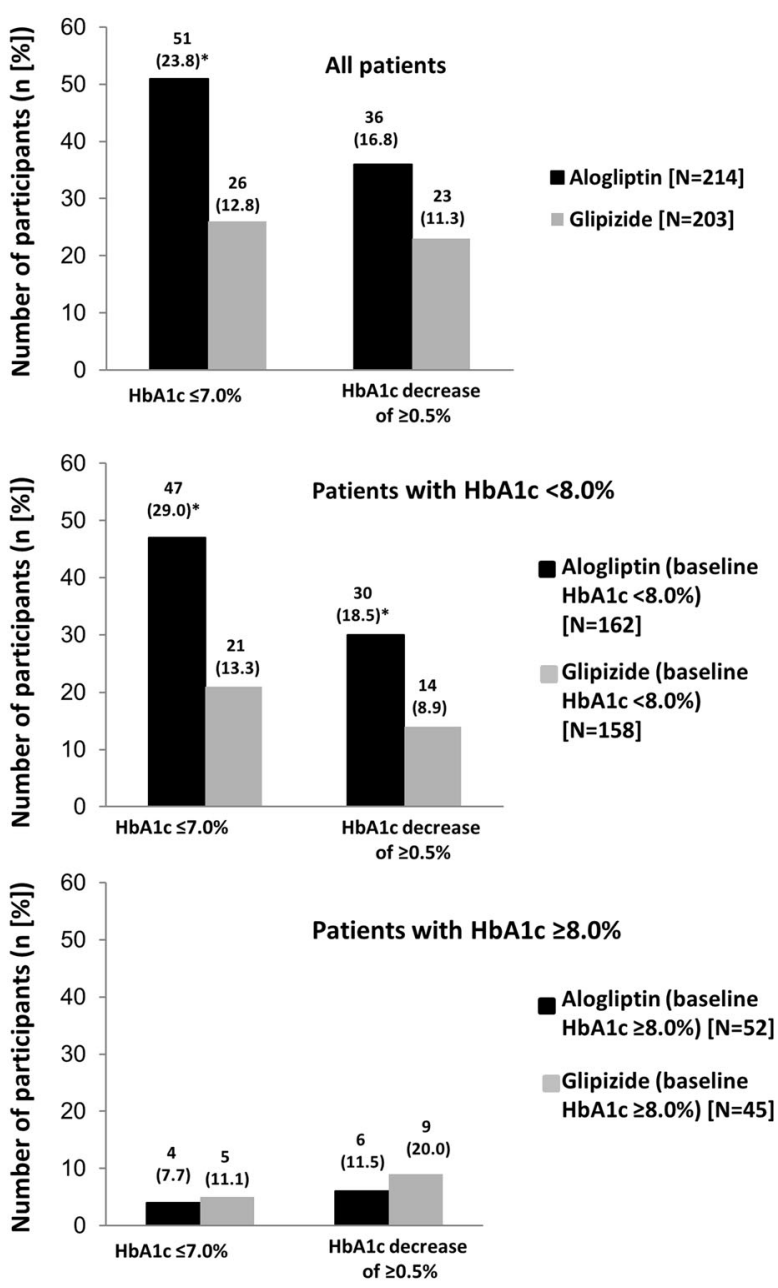

Fig. 3 Number of participants who achieved the composite criteria of no hypoglycemia and weight gain with either an $\mathrm{HbAlc}$ of $\leq 7.0 \%$ or an $\mathrm{HbAlc}$ decrease of $\geq 0.5 \%$ in a all patients, b patients with $\mathrm{HbAlc}<8.0 \%$, and c patients with $\mathrm{HbAlc} \geq 8.0 \%{ }^{*} p<0.03$ alogliptin versus glipizide. $H b A 1 c$ glycosylated hemoglobin

acceptably safe with significantly less hypoglycemia compared with glipizide; full tolerability data have been reported previously [12].

The assessment of HbA1c is a vital part of the clinical assessment of glycemic control. Most guidelines recommend a target HbA1c of $7.0 \%$, but emphasize the need to individualize treatment targets, especially in older patients with comorbidities [14]. Assessing other clinical outcomes, particularly safety, alongside HbA1c 


\begin{tabular}{lccc}
$\begin{array}{l}\text { Composite Criteria } \\
\text { No Hypoglycemia or } \\
\text { Weight Gain Plus }\end{array}$ & $\begin{array}{c}\text { Odds Ratio } \\
\text { Alogliptin } \\
\text { vs Glipizide }\end{array}$ & $95 \%$ Cl & p-valu \\
HbA1c $\leq 7.0 \%$ & 2.2 & $1.31,3.74$ & 0.003 \\
HbA1c decrease of $\geq 0.5 \%$ & 1.6 & $0.87,2.73$ & 0.135 \\
& & & \\
$\begin{array}{l}\text { HbA1c } \leq 7.0 \% \\
\text { (baseline HbA1c }<8.0 \%)\end{array}$ & 2.7 & $1.53,4.81$ & $<0.001$ \\
& & & \\
$\begin{array}{l}\text { HbA1c decrease of } \geq 0.5 \% \\
\text { (baseline HbA1c }<8.0 \%)\end{array}$ & 2.3 & $1.13,4.62$ & 0.021 \\
& & & \\
\hline $\begin{array}{l}\text { HbA1c } \leq 7.0 \% \\
\text { (baseline HbA1c } \geq 8.0 \% \text { ) }\end{array}$ & 0.6 & $0.13,2.64$ & 0.496 \\
$\begin{array}{l}\text { HbA1c decrease of } \geq 0.5 \% \\
\text { (baseline HbA1c } \geq 8.0 \%)\end{array}$ & 0.54 & $0.17,1.70$ & 0.290 \\
\hline
\end{tabular}

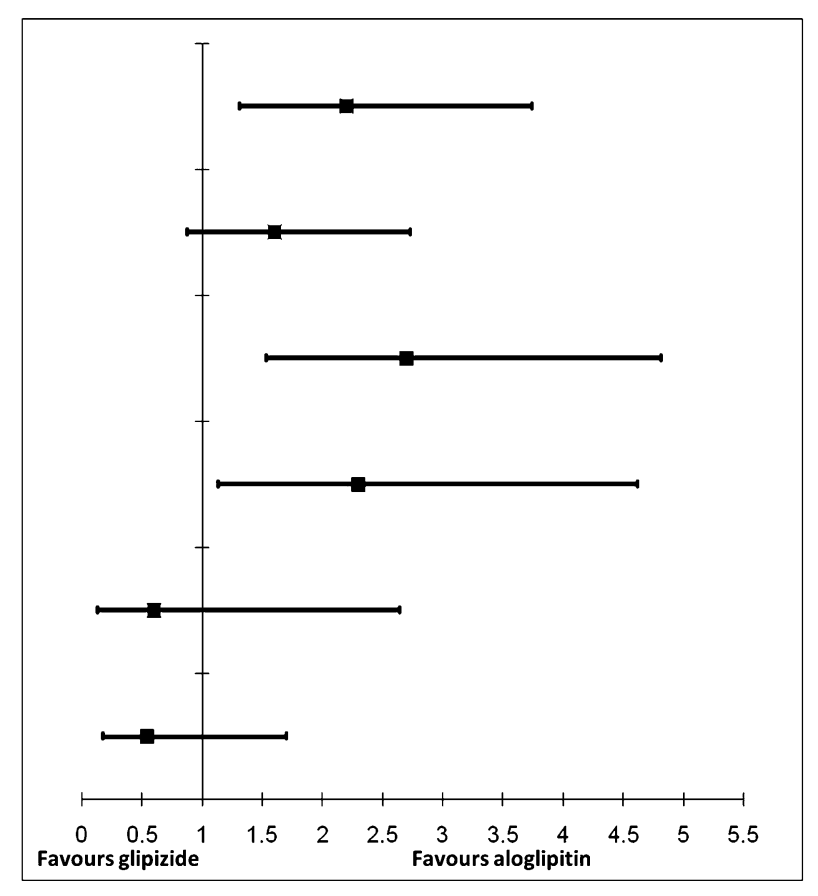

Odds ratio

versus glipizide. $C I$ confidence interval, $H b A I c$ glycosylated hemoglobin

Modified-Release Controlled Evaluation (ADVANCE) [16], and the Veterans Affairs Diabetes Trial (VADT) [17], have shown no reduction in primary cardiovascular endpoints with tight glycemic control when compared with standard glycemic control in older patients. Indeed, the ACCORD study showed a $22 \%$ increase in total mortality with the intensive therapy regimen in patients aged $40-79$ years. The cause of the unchanged or even negative effect of intensive therapy on cardiovascular endpoints is unclear but older age is a risk factor for severe hypoglycemia [18], and older patients are at greater risk of hypoglycemia-associated morbidity, which could be a contributory factor [19]. This increased risk of severe hypoglycemia is thought to be due to a combination of age- 
related comorbid conditions present in this population as well as a lack of awareness of the symptoms of hypoglycemia, such as cognitive dysfunction, weakness, and unsteadiness, which are often mistaken for other conditions. Hypoglycemia in this population can lead to significant morbidity, including serious vascular events such as stroke and myocardial infarction [19]. Therefore, avoiding —or at least reducing-hypoglycemia risk in the older patient is particularly important and will impart significant clinical benefits. Indeed, the importance of avoiding hypoglycemia in the elderly is uniformly recognized as a key feature in the treatment of this patient population. The recently updated Canadian Diabetes Association (CDA) guidelines recognize the importance of hypoglycemia avoidance over achieving glycemic targets in some elderly patients, including those who are frail or demonstrate cognitive impairment [20]. These guidelines suggest that, in these patients, sulfonylureas should be used with caution, further highlighting the need for individualized treatments.

Weight gain has been associated with increased cardiovascular risk in patients with T2DM and is, therefore, another important clinical outcome that should be carefully controlled [21]. The benefits of DPP-4 inhibitors in terms of weight neutrality and no increased risk of hypoglycemia have been recognized in the recent position statement published by the ADA and the European Association for the Study of Diabetes (EASD) [3]. The use of composite analyses, such as the ones used in this exploratory study, allows for the assessment of more than 1 clinically relevant outcome. This is important in the decision-making process for selecting treatment options for T2DM, particularly in the older patient, because often there is a need to focus on and consider more than 1 therapeutic goal. A number of studies comparing the efficacy of DPP-4 inhibitors and sulfonylureas on composite outcomes, comprising HbA1c targets, without hypoglycemia or weight gain have now been published. Similar to the results found in the current analysis, sitagliptin and saxagliptin, in addition to metformin, were found to result in more adult patients with T2DM achieving HbA1c targets without hypoglycemia or weight gain compared with glipizide [22, 23]. Vildagliptin and linagliptin have also been shown to help more T2DM adult patients inadequately controlled with metformin reach a composite endpoint of HbA1c $<7.0 \%$ with no hypoglycemia and no weight gain when compared with another sulfonylurea, glimepiride [24, 25]. Although these studies were all performed in adult patients and were not specifically conducted in older patient cohorts, one study [24] that examined the effectiveness of antidiabetic drugs in different age groups found that vildagliptin was superior to glimepiride in achieving an HbA1c target of $<7.0 \%$ with no hypoglycemia and no weight gain in patients of all ages, including those aged 60 to $<70$ and 70 to $<80$ years.

In our original study [12], alogliptin and glipizide resulted in similar reductions in HbA1c at Week 52 from baseline using the last observation carried forward (the primary endpoint), as well as reductions in observed HbA1c values throughout the 52-week period. However, in both groups, reductions from baseline to Week 52 in the primary endpoint were only small; a finding that was not predicted. In an exploratory analysis performed on the data from the per-protocol set in the original study, a comparison between patients who completed the study and those 
who received rescue medication was performed [12]. This analysis found that while patients who completed the study (i.e., who did not receive rescue medication) demonstrated reductions in HbA1c within the magnitude expected with alogliptin or glipizide, those who did receive rescue medication actually showed an increase in HbA1c levels. Therefore, it would appear that the inclusion of rescued patients, with the last observation carried forward, may have been a contributing factor to the unexpectedly small reductions in HbA1c from baseline to Week 52 [12]. A further possible explanation was provided by the post hoc analysis of drug-naïve versus washout patients. As observed in patients who completed the study, drug-naïve patients showed predicted reductions in their levels of HbA1c. However, patients entering the study after the 4-week washout period demonstrated a negligible change in HbA1c levels. Therefore, the relatively short washout period in patients who had already received antidiabetic monotherapy may have been a second contributory factor toward this unexpected finding.

The composite data from this current exploratory analysis further support the primary data previously published. In these data, the alogliptin group demonstrated considerably fewer hypoglycemic episodes and as well as significant body weight decreases, compared with the glipizide group [12]. Indeed, while there were small but significant weight reductions observed in the alogliptin group, weight increases were observed in the glipizide group [12]. These findings are important, particularly in older patients who are at risk of further complications associated with hypoglycemia.

Although the numbers of patients with a baseline HbA1c of $\geq 8.0 \%$ in the alogliptin and glipizide groups who achieved both composite endpoints were not significantly different, the sample sizes were very small, making the interpretation of results difficult. In addition, it is possible that patients with higher baseline HbA1c levels may be less responsive to treatment and therefore less likely to achieve HbA1c $\leq 7.0 \%$ without hypoglycemia or weight gain.

There are study limitations that warrant discussion. For example, the titration of glipizide was limited to $10 \mathrm{mg}$ in this population of patients. This was due to the age of the patient population, as older patients are more at risk of complications, including hypoglycemia, with higher doses of glipizide. Limiting the titration avoided any imbalances in hypoglycemia that could have been attributed to inappropriately high doses of glipizide in this older population. Another potential limitation relates to the post hoc nature of this report; this type of analysis is an appropriate exploratory examination of the primary data further supporting the initial findings, and one that is often performed after the initial study is complete. Lastly, although the results from this study provide important data on the treatment of older patients with T2DM, interpretation of the results must be limited to those between the ages of 65 and 90 years, as per the primary objective of the study.

Diabetes is a complex disease with many contributory factors. Treatments that can target more than one aspect of the condition will offer patients additional benefits. For example, in a systematic review and meta-analysis of 53 studies, 17 of which reported endpoint lipid levels, DPP-4 inhibitors were found to significantly reduce total cholesterol levels compared with controls [26]. Other studies have also demonstrated improvements in lipid levels with DPP-4 inhibitors [27-32], with these 
agents reportedly being more effective at reducing lipid levels than alpha glucosidase inhibitors [32]. Within class differences have been observed between the DPP-4 inhibitors, with alogliptin showing a greater reduction in total cholesterol and LDL levels than sitagliptin [28]. The efficacy of vildagliptin, sitagliptin and saxagliptin for reducing lipids varies, with one study showing vildagliptin to be more effective at reducing total cholesterol than sitagliptin and saxagliptin [30], and another showing vildagliptin to be more effective than sitagliptin at reducing total cholesterol and triglycerides [29]. Although further studies are still required, these differences between and within drug classes suggest some treatments may be of more value to certain populations of patients with T2DM than others, depending on their characteristics.

Sulfonylureas have been available for many years and the efficacy, safety and tolerability profile of this class of drug is well established. While DPP-4 inhibitors also have a good safety profile, the recent observation that linagliptin may result in liver toxicity demonstrates that additional findings are still being reported with this newer class of drugs [33]. When considering the value of individual treatments for different patient populations, both efficacy and safety should be considered. For example, elderly patients often have multiple comorbidities and although the well-established safety and tolerability profile of sulfonylureas is beneficial in this population, the risk of hypoglycemia with these agents may be of particular concern. In addition, cost also plays a part in the treatment decision. Cost is one of the main disadvantages of DPP-4 inhibitors [34]. This is particularly true in countries, such as Germany, where DPP-4 inhibitors are not reimbursed [35]. It is in these situations that value-based assessments are important. It is possible that, on balance, in elderly patients who are at high risk of hypoglycemia and may also have other comorbid conditions, DPP-4 inhibitors are likely to offer more value than the sulfonylureas. As such, more and more focus is being placed on personalized medicine in older patients with T2DM, addressing not only glycemic control but also other factors that might increase morbidity and mortality in this population [36-40]. In light of concerns that aggressively lowering HbA1c may be harmful in older patients with diabetes, especially those who are frail or have multiple comorbidities [15, 41], flexible glycemic targets are more desirable in this patient group. By examining composite endpoints that encompass not only indicators of efficacy but also issues of concern for both clinicians and patients, such as hypoglycemia and weight gain, the current analysis attempts to provide an insight into the overall success of glycemic control and its adverse effects in older patients. Such an approach may also offer a way of differentiating the various treatment options available for T2DM in older patients. Treatment options for T2DM are shown to have similar efficacy in terms of glycemic control; hence, additional clinical outcomes may add value and play a more important role. Data such as these can only help clinicians in getting closer to achieving a more personalized approach to T2DM treatment in this high-risk population of patients.

\section{CONCLUSION}

In conclusion, these analyses suggest that, in older patients aged 65-90 years with T2DM, alogliptin treatment can achieve a target HbA1c of $\leq 7.0 \%$ without hypoglycemia or weight gain in more patients than glipizide; these results particularly apply to the subpopulation of patients with baseline HbA1c below 8.0\%. 


\section{ACKNOWLEDGMENTS}

Sponsorship and article processing charges for this study were funded by Takeda Global Research \& Development Center, Inc., Deerfield, IL, and Takeda Global Research and Development Centre Ltd., London, United Kingdom. Manuscript writing and editorial assistance in the preparation of this manuscript was provided by Sarah Baldock, Gillian Gummer and Pin Lu (Rx Communications, UK), with funding from Takeda Pharmaceuticals North America, Inc. The sponsors were involved in the design, methods, analysis and preparation of this paper.

The authors wish to thank the patients and investigators at the 110 study sites across 15 countries for their participation in this global study. Thanks must also go to Hung Lam (Takeda Global Research \& Development Center, Deerfield, IL), who assisted with the statistical analyses for this study.

All named authors meet the ICMJE criteria for authorship for this manuscript, take responsibility for the integrity of the work as a whole, and have given final approval to the version to be published. Morgan Bron, Penny Fleck, and Craig Wilson contributed to the study design, analysis and interpretation of the data, and critical review of the manuscript.

Conflict of interest. Morgan Bron is an employee of Takeda Pharmaceuticals International, Inc., and owns granted stocks. Craig Wilson is an employee of Takeda Global Research \& Development Center, Inc. Penny Fleck is an employee of Takeda Global Research \& Development Center, Inc.

Compliance with ethics guidelines. The analysis in this article is based on previously conducted studies, and does not involve any new studies of human or animal subjects performed by any of the authors. The original study was performed in accordance with the Declaration of Helsinki, the International Conference on Harmonisation's Harmonised Tripartite Guideline for Good Clinical Practice, and all applicable regional laws and regulations. An institutional review board or ethics committee of each participating site conducted the initial approval and continuing review of the study and all patients signed an informed consent form prior to undergoing any procedures.

Open Access. This article is distributed under the terms of the Creative Commons Attribution Noncommercial License which permits any noncommercial use, distribution, and reproduction in any medium, provided the original author(s) and the source are credited.

\section{REFERENCES}

1. Percentage of civilian, noninstitutionalized population with diagnosed diabetes, by age, United States, 1980-2011. Centers for Disease Control and Prevention (online). Available at: http://www.cdc. gov/diabetes/statistics/prev/national/figbyage.htm. Accessed July 5, 2013.

2. Whiting DR, Guariguata L, Weil C, Shaw J. IDF Diabetes Atlas: global estimates of the prevalence of diabetes for 2011 and 2030. Diabetes Res Clin Pract. 2011;94:311-21.

3. Inzucchi SE, Bergenstal RM, Buse JB, et al. Management of hyperglycaemia in type 2 diabetes: a patient-centred approach. Position statement of the American Diabetes Association (ADA) and the European Association for the Study of Diabetes (EASD). Diabetes Care. 2012;35:1364-79.

4. Metformin $\mathrm{HCl}$ tablets [package insert]. Princeton, NJ: Bristol-Myers Squibb Company; 2006.

5. Pratley RE, Rosenstock J, Pi-Sunyer FX, et al. Management of type 2 diabetes in treatment-naïve elderly patients: benefits and risks of vildagliptin monotherapy. Diabetes Care. 2007;30:3017-22. 
6. Pratley RE, McCall T, Fleck PR, Wilson CA, Mekki Q. Alogliptin use in elderly people: a pooled analysis from phase 2 and 3 studies. J Am Geriatr Soc. 2009;57:2011-9.

7. DeFronzo R, Fleck P, Wilson C, Mekki Q, Alogliptin Study 010 Group. Efficacy and safety of the dipeptidyl peptidase-4 inhibitor alogliptin in patients with type 2 diabetes and inadequate glycemic control. Diabetes Care. 2008;31:2315-7.

8. Nauck M, Ellis G, Fleck P, Wilson CA, Mekki Q, Alogliptin Study 008 Group. Efficacy and safety of adding the dipeptidyl peptidase-4 inhibitor alogliptin to metformin therapy in patients with type 2 diabetes inadequately controlled with metformin monotherapy: a multicentre, randomised, double-blind, placebo-controlled study. Int J Clin Pract. 2009;63:46-55.

9. Pratley R, Kipnes M, Fleck P, Wilson C, Mekki Q, Alogliptin Study 007 Group. Efficacy and safety of the dipeptidyl peptidase-4 inhibitor alogliptin in patients with type 2 diabetes inadequately controlled by glyburide monotherapy. Diabetes Obes Metab. 2009;11:167-76.

10. Pratley R, Reusch J, Fleck P, Wilson CA, Mekki Q, Alogliptin Study 009 Group. Efficacy and safety of the dipeptidyl peptidase-4 inhibitor alogliptin added to pioglitazone in patients with type 2 diabetes: a randomized, double-blind, placebocontrolled study. Curr Med Res Opin. 2009;25: 2361-71.

11. Rosenstock J, Rendell M, Gross JL, Fleck PR, Wilson CA, Mekki Q. Alogliptin added to insulin therapy in patients with type 2 diabetes reduces $\mathrm{HbA}(1 \mathrm{C})$ without causing weight gain or increased hypoglycaemia. Diabetes Obes Metab. 2009;11: 1145-52.

12. Rosenstock J, Wilson C, Fleck P. Alogliptin versus glipizide monotherapy in mildly hypoglycaemic elderly patients with type 2 diabetes: a prospective, double-blind, randomized, 1-year study. Diabetes Obes Metab. 2013;15:906-14.

13. American Diabetes Association Workgroup on Hypoglycemia. Defining and reporting hypoglycemia in diabetes. Diabetes Care. 2005;28: 1245-9.

14. American Diabetes Association. Standards of medical care in diabetes-2011. Diabetes Care. 2011;34(Suppl 1):S11-61.

15. Action to Control Cardiovascular Risk in Diabetes Study Group, Gerstein HC, Miller ME, et al. Effects of intensive glucose lowering in type 2 diabetes. N Engl J Med. 2008;358:2545-59.
16. ADVANCE Collaborative group, Patel A, MacMahon $\mathrm{S}$, et al. Intensive blood glucose control and vascular outcomes in patients with type 2 diabetes. N Engl J Med. 2008;358:2560-72.

17. Duckworth W, Abraira C, Moritz T, et al. Glucose control and vascular complications in veterans with type 2 diabetes. N Engl J Med. 2009;360:129-39.

18. Zoungas S, Patel A, Chalmers J, For the ADVANCE collaborative group, et al. Severe hypoglycemia and risks of vascular events and death. N Engl J Med. 2010;363:1410-8.

19. Zamitt NN, Frier BM. Hypoglycemia in type 2 diabetes. Diabetes Care. 2005;28:2948-60.

20. Canadian Diabetes Association Clinical Practice Guidelines Expert Committee. Diabetes in the Elderly. Can J Diabetes. 2013;37(Suppl 1):S184-90.

21. Russell-Jones D, Khan R. Insulin-associated weight gain in diabetes-causes, effects and coping strategies. Diabetes Obes Metab. 2007;9:799-812.

22. Seck T, Engel SS, Williams-Herman DE, et al. Sitagliptin more effectively achieves a composite endpoint for A1C reduction, lack of hypoglycemia and no body weight gain compared with glipizide. Diabetes Res Clin Pract. 2011;93:e15-7.

23. Göke B, Gallwitz B, Eriksson JG, Hellqvist A, GauseNilsson I. Saxagliptin vs. glipizide as add-on therapy in patients with type 2 diabetes mellitus inadequately controlled on metformin alone: long-term (52-week) extension of a 52-week randomised controlled trial. Int $\mathrm{J}$ Clin Pract. 2013;67:307-16.

24. Bader G, Geransar P, Schweizer A. Vildagliptin more effectively achieves a composite endpoint of $\mathrm{HbA}_{1 \mathrm{c}}<7.0 \%$ without hypoglycaemia and weight gain compared with glimepiride after 2 years of treatment. Diabetes Res Clin Pract. 2013;100: e78-81.

25. Gallwitz B, Rosenstock J, Emser A, von Eynatten M, Woerle HJ. Linagliptin is more effective than glimepiride at achieving a composite outcome of target $\mathrm{HbA}_{1 \mathrm{c}}<7 \%$ with no hypoglycaemia and no weight gain over 2 years. Int J Clin Pract. 2013;67: 317-21.

26. Monami M, Lamanna C, Desideri CM, Mannucci E. DPP-4 inhibitors and lipids: systematic review and meta-analysis. Adv Ther. 2012;29:14-25.

27. Kubota A, Maeda H, Kanamori A, et al. Pleiotropic effects of sitagliptin in the treatment of type 2 diabetes mellitus patients. J Clin Med Res. 2012;4: 309-13. 
28. Kutoh E, Yamashita H. Differential effects on metabolic parameters between sitagliptin and alogliptin in drug naïve subjects with type 2 diabetes. J Diab Res Clin Metab. 2012. Available at: http://www.hoajonline.com/journals/pdf/20500866-1-17.pdf. Accessed Sept 24, 2014.

29. Choe EY, Cho Y, Choi Y, et al. The effect of DPP-4 inhibitors on metabolic parameters in patients with type 2 diabetes. Diabetes Metab J. 2014;38:211-9.

30. Saglietti G, Placentino G, Schellino A. Observational study on dipeptidyl peptidase-4 inhibitors: a real life analysis on 360 patients from the ASL VCO territory in Italy. Clin Drug Investig. 2014;34:513-9.

31. Shigematsu E, Yamakawa T, Kadanosono K, Terauchi Y. Effect of sitagliptin on lipid profile in patients with type 2 diabetes mellitus. J Clin Med Res. 2014;6:327-35.

32. Tsuchiya M. Alogliptin ameliorates dyslipidemia and LDL-size in patients with IGT or type 2 diabetes: comparing alogliptin and voglibose. Diabetes. 2011;60:A280.

33. Kutoh E. Probable linagliptin-induced liver toxicity: a case report. Diabetes Metab. 2014;40:82-4.

34. Davidson JA. The placement of DPP-4 inhibitors in clinical practice recommendations for the treatment of type 2 diabetes. Endocr Pract. 2013;19:1050-61.
35. International Diabetes Federation. Access to diabetes care in Europe: Country profiles. Germany. Available at: http://www.idf.org/sites/ default/files/GERMANY.pdf. Accessed Sept 24, 2014.

36. Hornick T. Managing diabetes in the elderly: go easy, individualize. Cleve Clin J Med. 2008;75:70-8.

37. Klonoff DC. Personalized medicine for diabetes. J Diabetes Sci Technol. 2008;2:335-41.

38. Rosenstock J. Management of type 2 diabetes mellitus in the elderly: special considerations. Drugs Aging. 2001;18:31-44.

39. Ismail-Beigi F, Moghissi E, Tiktin M, Hirsch IB, Inzucchi SE, Genuth S. Individualizing glycemic targets in type 2 diabetes mellitus: implications of recent clinical trials. Ann Intern Med. 2011;154: 554-9.

40. Pozzilli P, Leslie RD, Chan J, et al. The A1c and ABCD of glycaemia management in type 2 diabetes: a physician's personalized approach. Diabetes Metab Res Rev. 2010;26:239-44.

41. American Geriatrics Society Expert Panel on the Care of Older Adults with Diabetes Mellitus. Guidelines abstracted from the American Geriatrics Society Guidelines for improving the care of older adults with Diabetes Mellitus: 2013 update. JAGS. 2013;61:2020-6. 